\title{
18
}

\section{Internetworking of DQDB subnetworks: A maximum throughput analysis}

\author{
Andrzej R. PACH 1 \\ The University of Mining and Metallurgy \\ Al. Mickiewicza 30, 30-059 Krakow, Poland \\ tel. +48 12 345582, fax: +4812 342372, e-mail: pach@kt.agh.edu.pl \\ Sergio PALAZZO and Daniela PANNO2 \\ Universita' di Catania \\ V.le A . Doria 6, 95125 Catania, Italy \\ tel.+3995 339449, fax: +3995 338280,e-mail: palazzo@iit.unict.it
}

\begin{abstract}
This paper investigates the throughput performance of a system made up of a number of IEEE 802.6 subnetworks interconnected among each other, in overload conditions. The approach adopted in modelling the internet system enable us to leave any technical implementation issue regarding the Interconnection Unit (IU) out of consideration. In this respect, the IU is viewed as a black box characterized by some basic functionalities which may be met by a wide range of interface protocols. An analytical methodology is presented which allows us to solve some relevant design problems. In particular, we show that by non-linear programming algorithms it is possible to determine the best location and protocol of the IU interfaces on condition that the maximum system throughput is achieved. Finally, we provide some numerical examples concerning two identical DQDB subnetworks interconnected by IUs, located at either the middle or the headend of the subnetworks.
\end{abstract}

\section{Keywords}

Metropolitan area networks, DQDB, IEEE 802.6, bridges, internetworking

\footnotetext{
${ }^{1}$ This research was supported by the Polish Committee for Research under Grant no. 3 P406 00307.

${ }^{2}$ This research was partially supported by the Italian Ministry of University and Research under Grant MURST $60 \% 1993$.
} 


\section{INTRODUCTION}

The purpose of Metropolitan Area Networks is to provide integrated services over a large geographic area. In the past few years, substantial attention has been devoted to networks based on the IEEE 802.6 standard (IEEE, 90). An overview of major issues concerned with the modelling, analysis, and design of DQDB (Distributed Queue Dual Bus) networks described in the standard may be found in (Mukherjee and Bisdikian, 1992). A typical MAN will consist of either a single DQDB network or, in the most general case, a number of DQDB subnetworks interconnected by an Interconnection Unit (IU). Hereafter we will refer to such a scenario, as depicted in Figure 1.

An IEEE 802.6 subnetwork is based on a pair of unidirectional buses. The access of segments to the buses is controlled by the DQDB protocol, the basic operation of which we assume to be well-known to the reader. User information is transferred among nodes in segments accessing slots which are synchronously generated. In order to keep track of the traffic volume flows within the subnetwork each node is equipped with counters which count segments or requests (on the opposite bus) passing by. A reservation assignment determines univocally in which slot each segment will be transmitted.

Since future MANs will operate at very high speeds, IU operations should be very simple. For efficiency reasons, we assume that an IU operates at the Medium Access Control (MAC) layer and handles single segments rather than reassembled frames. The IU may be implemented in many ways, for example like a store-and-forward switch, an ATM switching network or a DQDB network. However, because the analysis presented in this paper is not affected by the specific IU implementation, we can consider the IU as a black box whose primary function is to recognise and route segments according their addresses.

When designing the interconnection of DQDB subnetworks, in addition to the problem to achieve a correct routing function, other problems may arise: for example, how to avoid subnetwork overloading due to the traffic generated in other subnetworks, especially in the case of different data transmission speeds on the buses; or, how to eliminate segments that have already found their destinations before reaching the IU, thus minimising the total delay and maximising the system throughput. The resolution of these problems requires a certain degree of interface complexity in terms of switching functions, buffering and resource allocation. In this sense, IUs for DQDB subnetworks are expected to have greater capabilities than classical bridges used in LANs, since they also have to actively control the traffic flows between subnetworks by the use of special signalling messages. In particular, in a DQDB internet environment, this can be implemented in a straightforward way by suitably forwarding requests to the overloading subnetworks. This kind of control may be used either to avoid a buffer overflow in the IU or to ensure equal shares of bandwidth among nodes on each bus of a subnetwork or in the whole system. In this respect, choice of an efficient interface protocol is of the greatest importance for the system performance.

Unfortunately, little attention has been devoted to the analysis and design of IUs. In (Spratt, 1991) it was demonstrated by simulation that a frame delay across bridged subnetworks is much less than the sum of the frame delays of the individual subnetworks. In (Dittmann and Tran-Gia, 1992) erasure nodes were proposed as an implementation of two-port bridges. The delay performance was investigated for different types of protocols implemented at erasure nodes. In (Khera et al., 1994) the effect of the bridge positioning on the behavioural characteristics of a hierarchical DQDB internet was discussed, mainly in terms of minimum 
average access delay. Finally, in (Nagano and Tasaka, 1994) the range of permissible positions for a DQDB gateway was determined in order to keep the loads on both buses at low levels as a performance requirement.

In this paper, we are concerned with the problem of determining the maximum system throughput in overload conditions for a general system of DQDB subnetworks interconnected by an IU. Throughout the analysis we adopt an approach similar to the one presented in (Pach et al., 1993), where a DQDB subnetwork with erasure nodes was dealt with. Here, we present a methodology which enables us to model a wide range of IU protocols and configurations in a DQDB internet environment, and to solve a number of relevant design problems.

The paper is organized as follows. In Section 2 we present the analytical model of the DQDB internet scenario we consider. Section 3 contains the formulation of some problems relevant to the system performance in terms of maximum throughput and addresses the solution methods which may be adopted. In Section 4 we introduce some numerical examples which illustrate how the presented model may be used. Finally, some conclusions are drawn.

\section{THE SYSTEM MODEL}

Let us consider the system depicted in Figure 1. It consists of $K$ subnetworks interconnected by an IU. Subnetwork $k$ contains $N_{k}$ nodes and it is divided by interface $I_{k}$ into two parts of $n_{k}$ and $N_{k}-n_{k}$ nodes, respectively. Traffic generated at all nodes is assumed to be of a single priority and asynchronous type; however, considerations presented in the following may rather straightforward be extended to multipriority environment as well as isochronous traffic. Moreover, although the topology presented in Figure 1 is of a star type, the approach presented in the paper may also be generalised to a mesh topology in which several IUs exist.

The main task assigned to the IU is the reliable transfer of segments between subnetworks according to their addresses. In general, we assume that the interface devices possess a set of capabilities, some of which may be enabled or not.

1 Interface $I_{k}(k=1,2, \ldots, K)$ has the capability to recognise the segment address and, consequently, to route segments to the IU or not.

2 Interface $I_{k}(k=1,2, \ldots, K)$ has the capability to buffer the segments incoming from the subnetwork $k$ and addressed to the IU and the ones in the opposite direction.

3 Interface $I_{k}(k=1,2, \ldots, K)$ has to capability to filter the subnetwork traffic. This is done by the use of the PSR (Previous Slot Received) bit in the ACF (Access Control Field) of the slot, which, according to the IEEE 802.6 standard, allows interface $I_{k}$ to recognise and release slots containing segments that have already passed all their destinations. Any node which receives a segment is obliged to set the PSR bit in the next slot and any incoming slot is buffered by interface $I_{k}$ until the PSR bit in the next slot is available. If this bit is set to one, it means that the slot being stored has already reached its destination node and, therefore, it is released. In such a way only segments addressed to nodes located beyond interface $I_{k}$ are allowed to pass through it.

4 Interface $I_{k}(k=1,2, \ldots, K)$ has the capability to pass the incoming requests through and/or generate additional requests in subnetwork $k$. The latter capability allows interface $I_{k}$ to actively throttle down the traffic generated in the subnetwork in order, for example, to reserve more slots for intersubnetwork traffic. 

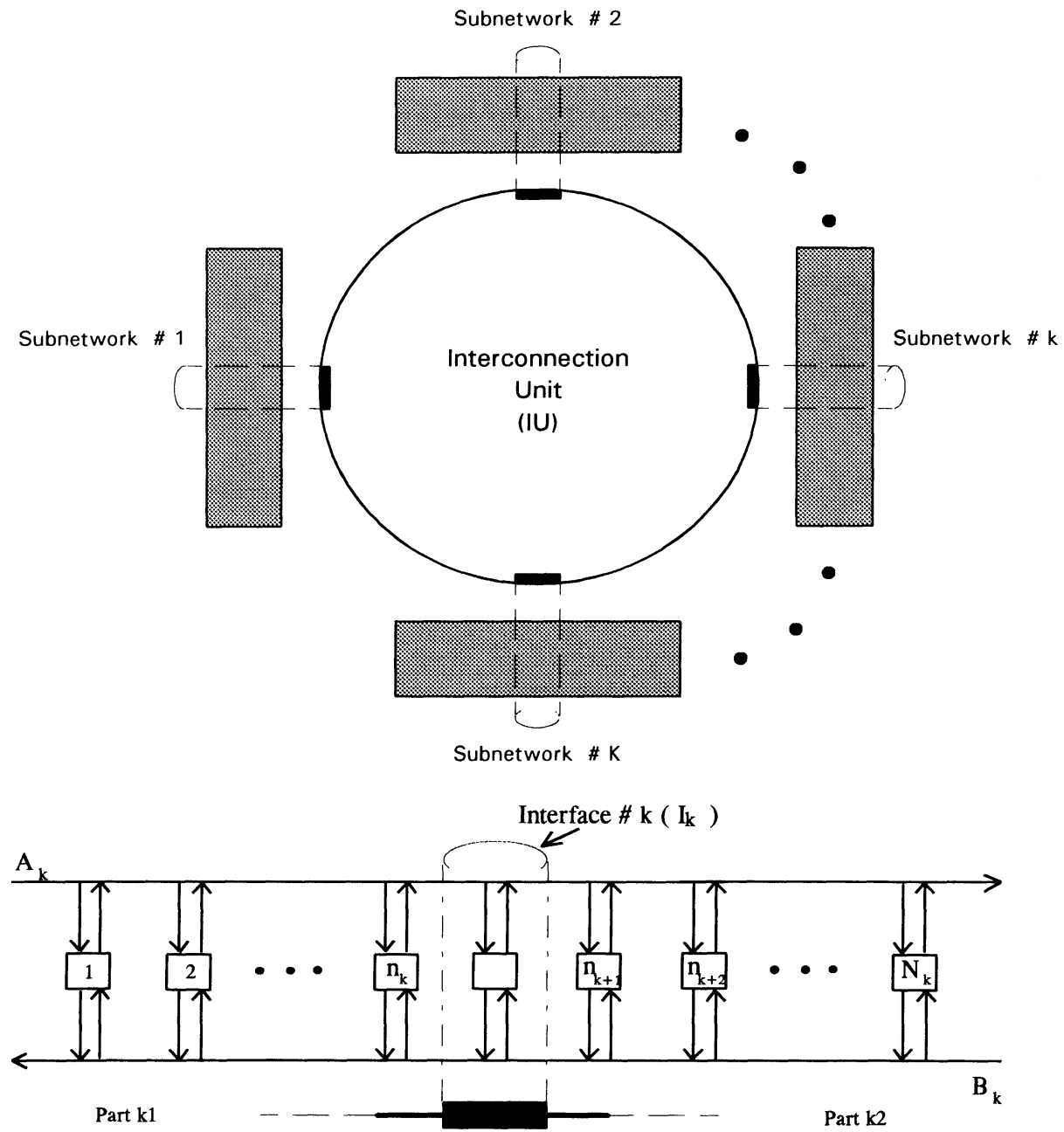

Figure 1 DQDB subnetworks linked by Interconnection Unit (IU).

According to which capabilities are enabled, interfaces may be implemented in several ways. As an example, we mention the following alternatives.

A Interface $I_{k}$ acts as a classical $D Q D B$ node. This means that, when there is a segment from the IU ready for transmission to subnetwork $k$, a request is transmitted. Interface $I_{k}$ is equipped with the request and countdown counters to determine in which slot the segment transmission should be undertaken. 
B Interface $I_{k}$ acts as an erasure $D Q D B$ node, in which the capability (3) is implemented. Then, in order to increase the subnetwork traffic a suitable protocol should be implemented to appropriately cancel or limit incoming requests from subnetwork $k$ or from the IU.

C Interface $I_{k}$ acts as a sort of insertion register node, which is able to erase slots containing segments previously read and transmits segments from the IU in released slots only.

Let us now introduce the following notation (all quantities are expressed in data units per time unit):

$C_{X, k}=$ capacity of Bus $X(X=\mathrm{A}$ or B $)$ in subnetwork $k$; we assume that $C_{A, k}=C_{B, k}=C_{k}$, $k=1,2, \ldots, K$;

$S_{X, n, k}=$ rate of segments transmitted on bus $X$ by node $n$ in subnetwork $k, X=\mathrm{A}, \mathrm{B}$, $k=1,2, \ldots, K, n=1,2, \ldots, N_{k}$

$R_{X, n, k}=$ rate of requests transmitted on bus $X$ by node $n$ in subnetwork $k, X=\mathrm{A}, \mathrm{B}$, $k=1,2, \ldots, K, n=1,2, \ldots, N_{k}$;

$T_{X, n, k}=$ available throughput (i.e. fraction of accessible data units) on bus $X$ at node $n$ in subnetwork $k, X=\mathrm{A}, \mathrm{B}, k=1,2, \ldots, K, n=1,2, \ldots, N_{k}$.

As follows from the basic DQDB operation, in the steady state, each segment transmission is accompanied by exactly one request transmission on the opposite bus, so:

$S_{X, n, k}=R_{Y, n, k}$

where $\mathrm{Y}$ indicates the opposite bus with respect to $\mathrm{X}$.

Let us now introduce an additional indexing to distinguish between parameters relevant to the two parts in which interface $I_{k}$ divides the subnetwork $k(k=1,2, \ldots, K)$ : conventionally, $k I$ will be reserved for the left-hand part whereas $k 2$ for the right-hand one, as shown in Figure 1 .

So, we denote by:

$\Lambda_{X, k m}$ the total intensity of the traffic generated in part $\mathrm{km}$ of subnetwork $k$ and transmitted on bus $X, X=\mathrm{A}, \mathrm{B}, m=1,2$.

$\alpha_{i m, j n}$ the fraction of segments generated in part im of subnetwork $i$ and passed through interface $I_{i}$ to part $j n$ of subnetwork $j, i, j=1,2, \ldots, K ; m, n=1,2$;

Observing that in the following we indicate with $\bar{n}$ the opposite part to $n$ in a subnetwork, we further denote by:

$\beta_{i n, \bar{n}}$ : the fraction of requests generated in the part in and passed through interface $I_{i}$ to part $i \bar{n}$ of the same subnetwork $i, i=1,2, \ldots, K ; n=1,2$.

$\beta_{i m, j n}$ the fraction of requests generated by interface $I j$ and transmitted to part $j n$ of subnetwork $j$, in order to reserve slots for traffic addressed from part im of subnetwork $i$ to part $j \bar{n}$ of subnetwork $j, i, j=1,2, \ldots, K ; i \neq j ; m, n=1,2$.

The available throughput on bus $\mathrm{A}$ at a node in a part of a subnetwork is equal to the bus capacity reduced by the segment traffic generated by other nodes within this part of the subnetwork and the request traffic on bus B originated from the opposite part or generated by the interface. The available throughput on bus B can be expressed in a similar way. So, we have that: 


$$
\begin{aligned}
& T_{A, n, k 1}=C_{k}-\sum_{\substack{i=1 \\
i \neq n}}^{n_{k}} S_{A, i, k 1}-\beta_{k 2, k 1} \Lambda_{A, k 2}-\sum_{\substack{j=1 \\
j \neq k}}^{K}\left(\beta_{j 1, k 1} \Lambda_{A, j 1}+\beta_{j 2, k 1} \Lambda_{B, j 2}\right) \quad n=1, \ldots, n_{k} \\
& T_{A, n, k 2}=C_{k}-\sum_{\substack{i=n_{k}+1 \\
i \neq n}}^{N_{k}} S_{A, i, k 2}-\alpha_{k 1, k 2} \Lambda_{A, k 1}-\sum_{\substack{j=1 \\
j \neq k}}^{K}\left(\alpha_{j 1, k 2} \Lambda_{A, j 1}+\alpha_{j 2, k 2} \Lambda_{B, j 2}\right) \quad n=n_{k}+1, \ldots, N_{k} \\
& T_{B, n, k 1}=C_{k}-\sum_{\substack{i=1 \\
i \neq n}}^{n_{k}} S_{B, i, k 1}-\alpha_{k 2, k 1} \Lambda_{B, k 2}-\sum_{\substack{j=1 \\
j \neq k}}^{K}\left(\alpha_{j 1, k 1} \Lambda_{A, j 1}+\alpha_{j 2, k 1} \Lambda_{B, j 2}\right) \quad n=1, \ldots, n_{k} \\
& T_{B, n, k 2}=C_{k}-\sum_{\substack{i=n_{k}+1 \\
i \neq n}}^{N_{k}} S_{B, i, k 2}-\beta_{k 1, k 2} \Lambda_{B, k 1}-\sum_{\substack{j=1 \\
j \neq k}}^{K}\left(\beta_{j 1, k 2} \Lambda_{A, j 1}+\beta_{j 2, k 2} \Lambda_{B, j 2}\right) \quad n=n_{k}+1, \ldots, N_{k}
\end{aligned}
$$

for $k=1,2, \ldots, K$.

Let us consider that the system is overloaded, i.e. all local node buffers always contain segments to be transmitted. Then, a problem of the fairness arises in that nodes obtain nonequal shares of the bandwidth. With respect of this, the current IEEE 802.6 standard defines the special mechanism called BandWidth Balancing (BWB) which aims at guaranteeing the steady state fairness in an individual DQDB subnetwork.

Let us assume that the BWB mechanism is enabled and the BWB modulus is $M_{X, \mathrm{~km}}$ for transmission on bus $X$ in part $k m$ of subnetwork $k, X=\mathrm{A}, \mathrm{B}, k=1,2, \ldots, K, m=1,2$. The mechanism forces each node to pass a fraction of $1 /\left(M_{X, k m}+1\right)$ accessible slots untouched and, therefore, node $n$ in part $m$ of subnetwork $k$ uses $M_{X, k m} /\left(M_{X, k m}+1\right)$ of the available throughput on bus $X$ as a maximum (Hahne et al., 1992). So

$S_{X, n, k m}=\frac{M_{X, k m}}{M_{X, k m}+1} T_{X, n, k m}$

By substituting this into (1), we get $4\left(N_{1}+N_{2}+\ldots+N_{K}\right)$ linear equations. Solving these equations we obtain that $S_{X, n, k m}=S_{X, k m}, X=\mathrm{A}, \mathrm{B}, n=1,2, \ldots, N_{K}, k=1,2, \ldots, K$. From this, we can derive that in any part of the interconnected subnetworks, with BWB mechanism enabled and under heavy loads, the rates of segments transmitted by nodes are the same regardless of the segment or requests traffic routed from the outside to the subnetwork. One can simply explain this by noting that any node in a given part of a subnetwork observes the same external traffic.

After some algebraic manipulations, and observing that $\Lambda_{X, k 1}=n_{k} S_{X, k 1}$, $\Lambda_{X, k 2}=\left(N_{k}-n_{k}\right) S_{X, k 2}$, we finally get the following set of linear equations: 


$$
\begin{aligned}
& \frac{M_{A, k 1} n_{k}+1}{M_{A, k 1}} S_{A, k 1}+\beta_{k 2, k 1}\left(N_{k}-n_{k}\right) S_{A, k 2}+\sum_{\substack{j=1 \\
j \neq k}}^{K}\left[\beta_{j 1, k 1} n_{j} S_{A, j 1}+\beta_{j 2, k 1}\left(N_{j}-n_{j}\right) S_{B, j 2}\right]=C_{k} \\
& \frac{M_{A, k 2}\left(N_{k}-n_{k}\right)+1}{M_{A, k 2}} S_{A, k 2}+\alpha_{k 1, k 2} n_{k} S_{A, k 1}+\sum_{\substack{j=1 \\
j \neq k}}^{K}\left[\alpha_{j 1, k 2} n_{j} S_{A, j 1}+\alpha_{j 2, k 2}\left(N_{j}-n_{j}\right) S_{B, j 2}\right]=C_{k} \\
& \frac{M_{B, k 1} n_{k}+1}{M_{B, k 1}} S_{B, k 1}+\alpha_{k 2, k 1}\left(N_{k}-n_{k}\right) S_{B, k 2}+\sum_{\substack{j=1 \\
j \neq k}}^{K}\left[\alpha_{j 1, k 1} n_{j} S_{A, j 1}+\alpha_{j 2, k 1}\left(N_{j}-n_{j}\right) S_{B, j 2}\right]=C_{k} \\
& \frac{M_{B, k 2}\left(N_{k}-n_{k}\right)+1}{M_{B, k 2}} S_{B, k 2}+\beta_{k 1, k 2} n_{k} S_{B, k 1}+\sum_{\substack{j=1 \\
j \neq k}}^{K}\left[\beta_{j 1, k 2} n_{j} S_{A, j 1}+\beta_{j 2, k 2}\left(N_{j}-n_{j}\right) S_{B, j 2}\right]=C_{k}
\end{aligned}
$$

for $k=1,2, \ldots, K$.

These equations fully describe the steady state traffic behaviour of $K$ subnetworks, with BWB mechanism enabled and under the overload conditions, when they are interconnected by an IU. The above set of equations may be solved to obtain segment rates $S_{A, k m}$ and $S_{B, k m}, k=$ $1,2, \ldots, K, m=1,2$. Having these rates, one can easily determine the total system throughput, $S$, as:

$$
S=\sum_{k=1}^{K}\left[n_{k}\left(S_{A, k 1}+S_{B, k 1}\right)+\left(N_{k}-n_{k}\right)\left(S_{A, k 2}+S_{B, k 2}\right)\right]
$$

\section{DESIGN ISSUES IN BRIDGING DQDB SUBNETWORKS}

Equations (2) and (3) allow us to solve some basic issues that we may encounter when analysing the system.

To begin with, let us point out that the presented analysis is valid for the steady state and when the system is overloaded, i.e. all nodes have always segments to be transmitted on both buses. Such an extreme situation may exceptionally happen in real systems. However, investigation of the system behaviour under such conditions may give some insight when the system is close to the saturation. Such an approach is commonly used in the analysis of individual DQDB subnetworks, where attention has mainly been paid to the throughput maximisation and the fairness of the system. It seems that overload analysis is particularly attractive for interconnected DQDB subnetworks too, since in that case designers should take into account the worst conditions in searching for optimal interface protocol and configurations. In a system consisting of a number of subnetworks interconnected by an IU we have some freedom in tuning the parameters $\alpha_{i m, j n}, \beta_{i n, \bar{n}}, \beta_{m, j n, i}$ and the BWB moduli $M_{X, k m}$ as well as the locations of interfaces $I_{k}$.

With respect to the last item, it is worth reminding that equations (2) and (3) have been derived for the case of one interface per subnetwork. It is possible to consider more complex topologies in which there are many IUs and more than one interface per subnetwork at the expense of some complexity in equations. Restricting ourselves to the configuration presented in Figure 1, the question is in which places in the subnetworks interfaces should be located. 
Another issue is according to which protocols interfaces should operate. Some implementation possibilities in this regard have been mentioned in the previous section.

Any protocol variant may conveniently be modelled by a proper definition of the relations between parameters $\alpha_{i m, j n}$ and $\beta_{i m, j n}$. For example, when interface $I_{k}$ acts as a classical DQDB node, then a request should be sent for each transmission of a segment (either originated in subnetwork $k$ or transferred from other subnetworks). In this case:

1. All traffic generated in one part of the subnetwork $i$ and transmitted on the bus is passed through by interface $I_{i}$ to the opposite part independently of the actual destination, that is $\alpha_{i n, \bar{n}}=1$.

2. Each request issued to reserve a slot for transmitting a segment within the same subnetwork part is passed through by interface $I_{i}$, that is $\beta_{i n, \bar{n}}=1$.

3. The transmission of a segment coming from another subnetwork is accompanied by the transmission, on the opposite bus, of a request generated by interface $I_{k}$, that is $\alpha_{j m, k \bar{n}}=$ $\beta_{j m, k n}, j, k=1,2, \ldots, K, j \neq k, m, n=1,2$.

Now, we address some basic problems which can be solved using the system model presented in the previous section.

To this purpose, let us introduce the following additional notation:

$\boldsymbol{P}_{\boldsymbol{k}}$ - protocol which is implemented at interface $I_{k}$;

$\boldsymbol{N}=\left\{n_{1}, n_{2}, \ldots, n_{K}\right\}$ - arrangement of interfaces in the system, where $n_{k}, k=1,2, \ldots \mathrm{K}$, is the number of the node which immediately precedes interface $I_{k}$;

$\boldsymbol{A}$ - distribution probability matrix of the segment destinations.

Our main objective is to find the best localisations for interfaces $I_{k}(k=1,2, \ldots, K)$ in terms of the system throughput maximisation. It is clear that the system performance is strongly influenced by the matrix $\mathrm{A}$.

If it is possible to install an interface in any place along the subnetwork, then the problem becomes:

\section{Problem 1:}

$$
\begin{gathered}
\text { given: } N_{1}, N_{2}, \ldots, N_{K}, \\
C_{1}, C_{2}, \ldots, C_{K}, \\
\boldsymbol{P}_{\mathbf{1}}, \boldsymbol{P}_{\mathbf{2}}, \ldots, \boldsymbol{P}_{\boldsymbol{K}} \text {, and } \\
\boldsymbol{A}
\end{gathered}
$$

maximise: $\boldsymbol{S}$ over all possible arrangements of $\boldsymbol{N}$.

However, in real situations some constraints concerning localisation of interfaces may be encountered; this is due to geographical reasons, available cabling as well as potential costs of interface placement. In this case, given the interface arrangement, we may consider another design task: to find a set of protocols that maximises the system throughput. As we mentioned before, this results in determining values of the parameters $\alpha_{i m, j n}$ and $\beta_{i m, j n}$ or relations between them. Then, the problem becomes: 


\section{Problem 2:}

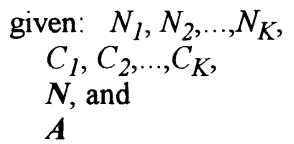

maximise: $\boldsymbol{S}$ over all possible sets $\left\{\boldsymbol{P}_{1}, \boldsymbol{P}_{2}, \ldots, \boldsymbol{P}_{K}\right\}$

Last, the combination of the problem presented above may be considered.

\section{Problem 3:}

$$
\begin{gathered}
\text { given: } N_{1}, N_{2}, \ldots, N_{K}, \\
C_{1}, C_{2}, \ldots, C_{K}, \text { and } \\
\boldsymbol{A}
\end{gathered}
$$

maximise: $\boldsymbol{S}$ over all possible $N$ and $\left\{\boldsymbol{P}_{1}, \boldsymbol{P}_{2}, \ldots, \boldsymbol{P}_{K}\right\}$

All problems presented above, however, might be considered with some specific constraints or performance requirements; for example, we can solve any of the above mentioned problems given that the fairness on both buses in each subnetwork is preserved.

The problems stated above, in general, do not have solutions in closed form except for very simple $I_{k}$ implementations and arrangements in the system. However, very often the optimal solutions can be easily found on a numerical way.

\section{NUMERICAL EXAMPLES}

In this section we present some simple numerical examples which illustrate the model and the problems previously introduced. As was discussed, in the general case, the expression for the system throughput can always be obtained in a closed form but, unfortunately, it may be very complex. Hence, in the following, we refer to the case of two identical subnetworks interconnected by an IU in order to show analytical results in simple closed forms. We consider two interface arrangements: interfaces are either located i) in the middle of subnetworks or ii) at their headends. We also assume that

$C_{1}=C_{2}=C$,

$N_{1}=N_{2}=2 N$,

$M_{X, k n}=M_{X, k \bar{n}}, X=\mathrm{A}, \mathrm{B}, k=1,2, n=1,2$.

A Interface $I_{1}$ and $I_{2}$ are located in the middle of subnetworks 1 and 2, respectively

In this case we have the interface arrangement $N=\left\{n_{1}=N ; n_{2}=N\right\}$. Let us further assume that the volumes of traffic interchanged between the parts of the two subnetworks are the same, that is: 


$$
\begin{array}{ll}
\alpha_{k n, k \bar{n}}=\alpha_{L} & k=1,2 \quad \text { and } n=1,2 \\
\alpha_{j m, k n}=\alpha_{E} & j, k=1,2(j \neq k) \quad \text { and } \quad n, m=1,2
\end{array}
$$

Due to the assumptions made on the identity of the subnetworks and the symmetry of the traffic, the equations (2) are the same for the two subnetworks. Moreover, from a simple reasoning on the symmetry of the interface arrangements we obtain:

$S_{A, 11}=S_{B, 12}=S_{A, 21}=S_{B, 22}=S_{1}$

$S_{B, 11}=S_{A, 12}=S_{B, 21}=S_{A, 22}=S_{O}$.

where with the index I (Inwards) we refer to the bus portions oriented toward the interface and with the index $O$ (Outwards) the opposite ones.

In the following we consider two protocols as possible variants to be implemented at the interfaces. It is assumed that the same protocol is incorporated in both interfaces.

\section{A.l Protocol $P_{1}$ : each interface behaves like a classical $D Q D B$ node}

In order to reserve a slot, each intersubnetwork segment transmission is accompanied by a request transmission on the opposite bus. Requests are generated at the interfaces and directed to the proper bus. Local requests (generated into the subnetwork at which the interface belongs) are passed through without any interface intervention. Each interface is equipped with the request and countdown counters to determine in which slot a intersubnetwork segment is allowed to be transmitted. With respect to parameters $\alpha_{i m, j n}$ and $\beta_{i m, j n}$, such an interface protocol can be described as follows:

$$
\begin{array}{ll}
\alpha_{k n, k \bar{n}}=\beta_{k n, k \bar{n}}=1 & k=1,2 \quad \text { and } n=1,2 \\
\alpha_{j m, k n}=\beta_{j m, k \bar{n}}=\alpha_{E} & j, k=1,2(j \neq k) \quad \text { and } n, m=1,2
\end{array} .
$$

In this case the set of equations (2) is reduced to four identical equations. The system is totally fair and all nodes in the system transmit segments with the same rate:

$$
S_{I}=S_{O}=S_{X, k m}=\frac{C M}{2\left(1+\alpha_{E}\right) M N+1} \quad X=A, B, k, m=1,2 .
$$

From (3) the maximum system throughput under protocol $\boldsymbol{P}_{1}$ is:

$$
S_{P_{1}}=8 N S_{X, k m}=\frac{8 C M N}{2\left(1+\alpha_{E}\right) M N+1} .
$$

Rather obviously, it can be observed that the system throughput decreases when $\alpha_{E}$ increases, i.e. when the intersubnetwork traffic increases. 
A.2 Protocol $P_{2}$ : each interface releases slots; the intersubnetwork segments are transmitted in any released slots.

Through the use of the PSR bit, the slots containing segments which previously found their destinations are released. The interface transmits intersubnetwork segments in the earliest available slots released, without any signalling through requests. Segments addressed from one subnetwork part to the other as well as local requests are passed through by the interface without being modified. Protocol $\boldsymbol{P}_{2}$ may be described as follows:

$$
\begin{aligned}
& \alpha_{k n, k \bar{n}}=\alpha_{L} \quad \text { and } \quad \beta_{k n, k \bar{n}}=1 \quad k=1,2 \quad \text { and } n=1,2 \\
& \alpha_{j m, k n}=\alpha_{E} \quad \text { and } \beta_{j m, k \bar{n}}=0 \quad j, k=1,2(j \neq k) \quad \text { and } n, m=1,2
\end{aligned}
$$

In this case we have:

$$
\begin{aligned}
& S_{I}=\frac{C M}{(M N+1)^{2}-\left(\alpha_{L}+2 \alpha_{E}\right) M^{2} N^{2}} \\
& S_{O}=\frac{C M\left[\left(1-\alpha_{L}-2 \alpha_{E}\right) M N+1\right]}{(M N+1)^{2}-\left(\alpha_{L}+2 \alpha_{E}\right) M^{2} N^{2}}
\end{aligned}
$$

and the maximum system throughput under protocol $\boldsymbol{P}_{2}$ is

$$
S_{P_{2}}=4 N\left(S_{I}+S_{O}\right)=\frac{4 C M N\left[2+\left(1-\alpha_{L}-2 \alpha_{E}\right) M N\right]}{(M N+1)^{2}-\left(\alpha_{L}+2 \alpha_{E}\right) M^{2} N^{2}} \text {. }
$$

We may observe that the system under protocol $\boldsymbol{P}_{2}$ exhibits unfairness within a subnetwork part: a node can transmit on a bus faster than on the other one. Fairness is only possible in the case when $\alpha_{L}+2 \alpha_{E}=1$.

Being $\Lambda_{X, k m}=\alpha_{I} \Lambda_{X, k m}+\alpha_{L} \Lambda_{X, k m}+2 \alpha_{E} \Lambda_{X, k m}$, where $\alpha_{I}$ represents the fraction of traffic generated and addressed inside the same subnetwork part, the above fairness condition is met only if $\alpha_{I}=0$.

\section{$B$ Interfaces $I_{1}$ and $I_{2}$ are located at the right headends of subnetworks 1 and 2 , respectively}

In this case, we have that $N=\left\{n_{l}=N_{1}=2 N, n_{2}=N_{2}=2 N\right\}$. Assuming symmetry in the traffic exchanged between subnetworks, from (2) we obtain two equations that fully describe the system. They are as follows: 
$\frac{2 M N+1}{M} S_{I}+2 \beta_{E} N S_{I}=C$

$2 \alpha_{E} N S_{1}+\frac{2 M N+1}{M} S_{O}=C$

where $\alpha_{E}=\alpha_{11,21}=\alpha_{21,11}$ and $\beta_{E}=\beta_{11,21}=\beta_{21,11}$.

\section{B.I Protocol $P_{1}$}

In this case each intersubnetwork segment transmission is accompanied by a request transmission, so we have that $\beta_{E}=\alpha_{E}$. Then, we obtain results which are identical to those presented for the configuration A. So, in the case of two identical subnetworks with symmetric traffic exchange and interfaces implemented as classical DQDB nodes, it makes no difference where these interfaces are located.

\section{B.2 Protocol $P_{2}$}

In this case $\beta_{E}=0$, and we obtain:

$$
\begin{aligned}
& S_{I}=\frac{C M}{2 M N+1} \\
& S_{O}=\frac{C M\left[2\left(1-\alpha_{E}\right) M N+1\right]}{(2 M N+1)^{2}}
\end{aligned}
$$

The maximum system throughput is:

$$
S_{P_{2}}=\frac{8 C M N\left[\left(2-\alpha_{E}\right) M N+1\right]}{(2 M N+1)^{2}} \text {. }
$$

Analogously to the case A.2, we observe that there may be an unfairness phenomenon However, in contrast to A.2, fairness can be achieved if $\alpha_{E}=0$, that is, when there is no intersubnetwork traffic.

In Table 1 we present the results obtained for the arrangements and protocols mentioned above, in the case of two identical subnetworks and symmetric traffic flows between subnetwork parts. The system parameters are given in the header of the table.

We can draw some observations from this table.

1 If protocol $\boldsymbol{P}_{\boldsymbol{1}}$ is implemented, there is no restriction on the interface positioning. The system exhibits the fairness, however, this is at the cost a certain degradation in the maximum system throughput in comparison with protocol $\boldsymbol{P}_{\mathbf{2}}$.

2 Protocol $\boldsymbol{P}_{2}$ offers a greater maximum throughput over a wide range of aunder arrangement $A$ than under arrangement $B$, but at the expense of a stronger unfairness. With 
respect to this, under arrangement $\mathrm{A}$ protocol $\boldsymbol{P}_{\mathbf{2}}$ exhibits an extreme unfairness at low values of $\alpha$. To explain it, let us refer to the limiting situation in which $\alpha=0$, i.e. all traffic generated in a subnetwork part finds its destination before reaching the interface. According to the protocol $\boldsymbol{P}_{2}$, the interface releases all slots on the inward bus but it exercises no control on the flood of requests passing through it on the opposite bus. This results in that the nodes are not able to transmit their segments on the inward bus $\left(S_{I} \approx 0\right)$.

Table 1 Node transmission rates and maximum system throughput for different interface arrangements and protocols

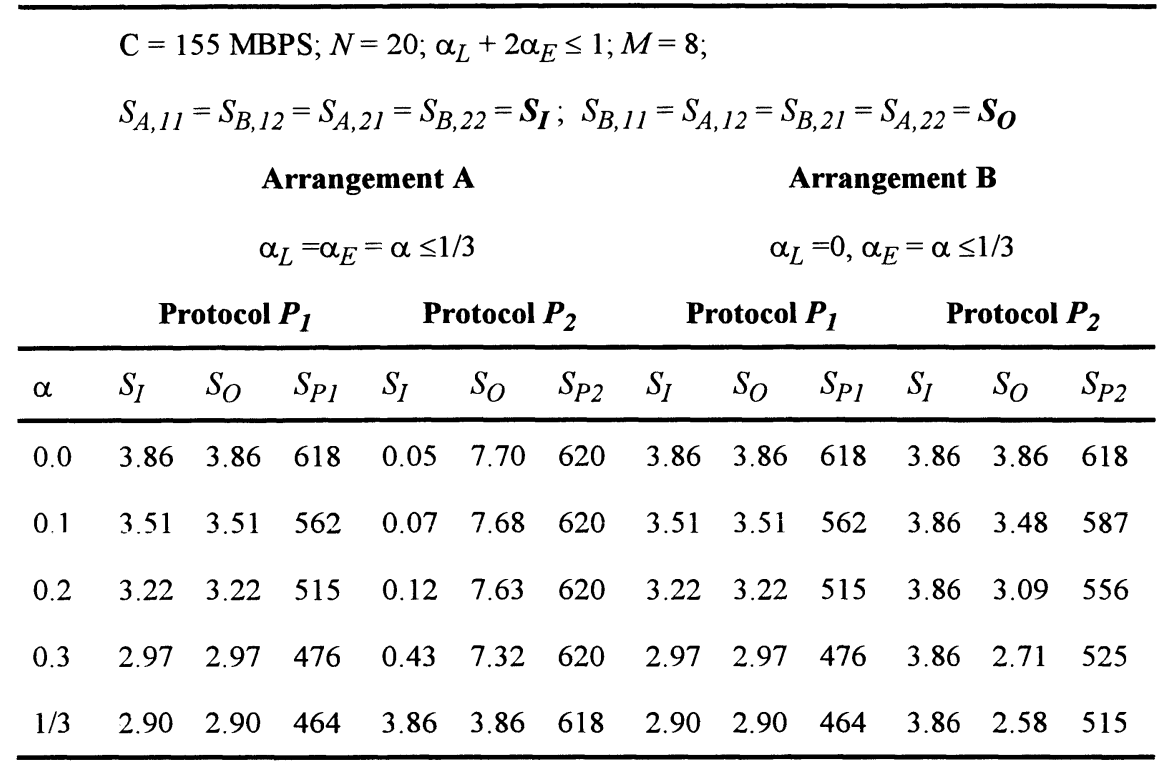

\section{CONCLUSIONS}

In this paper we presented an analytical model of a DQDB internet system in the case of overload conditions. As a matter of fact, DQDB subnetworks may be interconnected in several ways. So, we modeled the Interconnection Unit (IU) through a device that is only characterized by some basic functionalities which may be met by a wide range of interface protocols. In such a way we were able to determine the maximum throughput of the system, regardless of any technical issue relevant to the specific IU implementation. Then, we introduced some non-linear programming problems relative to selecting the proper locations and protocols of the IU interfaces, on condition that the maximum system throughput is achieved. Finally, we provided some numerical examples concerning two identical DQDB subnetworks interconnected by IUs, which are located at either the middle or the headend of the subnetworks and are characterized by two alternative interface protocols. These examples 
enabled us to point out that the IU interface position and protocol may sensibly influence the system behaviour in terms of maximum throughput and fairness.

\section{REFERENCES}

Dittmann, R. and Tran-Gia, P. (1992) Architecture alternatives and performance issues in DQDB subnetwork interconnection. Proc. EFOC/LAN 92, Paris.

Hahne, E.L., Choudhury, A., and Maxemchuk, N.F. (1992) Improving the fairness of DQDB networks. IEEE Trans. Commun., 40, 1192-1204.

IEEE Standard 802.6 (1990) Distributed Queue Dual Bus (DQDB): Subnetwork of a Metropolitan Area Network, Final Draft DIS.

Khera, H., Ilyas, M. and Bemmel V. (1994) Behavioral characteristics of DQDB subnetworks in a hierarchical communication system. Proc. ICC '94, New Orleans, LA.

Mukherjee B. and Bisdikian C. (1993) A journey through the DQDB network literature. Performance Evaluation, 16, 129-158.

Nagano, H. and Tasaka, S. (1994) The effect of the gateway location on the performance of DQDB networks. Proc. ICC '94, New Orleans, LA.

Pach, A.R., Palazzo,S., and Panno, D. (1993) Overload throughput performance of different erasure node protcols for DQDB networks. Proc. 18th Conf. on Local Area Networks, Minneapolis, $\mathbf{M N}$.

Spratt, M.P. (1991) Bandwidth allocation over several networks with DQDB. Proc. EFOC/LAN 91, London.

\section{BIOGRAPHIES}

Andrzej R. Pach received the M.Sc. degree in electrical engineering and the Ph.D. degree in telecommunications from the University of Mining and Metallurgy, Cracow, Poland, in 1976 and 1979, respectively, and the Ph.D.Hab. degree in telecommunications and computer networks from the Warsaw University of Technology in 1989.

In 1979, he joined the Telecommunications Department at the University of Mining and Metallurgy, where he is currently a Professor. From 1986 to 1987, he worked at CNET, France, as an International Scientific Consultant. In 1991, he spent his sabbatical leave at the University of Catania, Italy. His research interests include multiple access issues in communication networks, network interconnection, performance evaluation of lightweight and high-speed LAN/MAN protocols, and the design of broadband networks. He served as a secretary to the ITC Specialists' Seminar in 1991.

Sergio Palazzo received the degree in electrical engineering from the University of Catania, Italy, in 1977.

Until 1981, he was at ITALTEL, Milano, where he was involved in the design of operating systems for electronic exchanges. He then joined CREI, which is the center of the Politecnico di Milano for research on computer networks. Since 1987, he has been Associate Professor of Telecommunication Networks at the university of Catania. He served on the Technical Program Committees of the INFOCOM ' 92 conference and the Fifth IEEE Workshop on 
Metropolitan Area Networks. Presently, his research interests include broadband networks, MAN architectures and protocols, network management, and multimedia applications.

Daniela Panno received the degree in electrical engineering and the Ph.D. degree in telecommunications from the University of Catania, Italy, in 1989 and 1993, respectively.

She is now a Research Assistant at the Department of Informatics and Telecommunications, University of Catania. Her main interests are focused on protocols for metropolitan area networks and traffic control in ATM networks. 